\title{
Sciendo
}

Int. J. of Applied Mechanics and Engineering, 2018, vol.23, No.4, pp.897-910

DOI: 10.2478/ijame-2018-0050

\section{THERMAL DISTURBANCES IN TWINNED ORTHOTROPIC THERMOELASTIC MATERIAL}

\author{
L. RANI* and V. SINGH \\ School of Basic and Applied Sciences, Galgotias University \\ Greater Noida, U.P, INDIA \\ E-mail: Leena_kuk@rediffmail.com
}

\begin{abstract}
This paper deals with deformation in homogeneous, thermally conducting, single-crystal orthotropic twins, bounded symmetrically along a plane containing only one common crystallographic axis. The Fourier transforms technique is applied to basic equations to form a vector matrix differential equation, which is then solved by the eigen value approach. The solution obtained is applied to specific problems of an orthotropic twin crystal subjected to triangular loading. The components of displacement, stresses and temperature distribution so obtained in the physical domain are computed numerically. A numerical inversion technique has been used to obtain the components in the physical domain. Particular cases as quasi-static thermo-elastic and static thermoelastic as well as special cases are also discussed in the context of the problem.
\end{abstract}

Key words: generalized thermoelasticity, orthotropic material, relaxation time, Fourier transforms, Instantaneous loading.

\section{Introduction}

The classical uncoupled theory of thermoelasticity predicts two phenomena not compatible with the physical observations. First, the equation of heat conduction of this theory does not contain any elastic terms; second, the heat equation is of a parabolic type, predicting infinite speeds of propagation for heat waves. The coupling between the strain and temperature fields was first postulated by Duhamel [1] who derived equations for the distribution of strains in an elastic medium subjected to temperature gradients. Duhamel introduced the dilatation term in the equation of thermal conductivity but his equation was not on a thermodynamically satisfactory basis. Neumann [2], Voigt [3] and Jeffreys [4] made attempts at thermodynamical justification of equations of Duhamel's theory and solved a number of interesting problems.

Biot [5] gave a satisfactory derivation of the equation of thermal conductivity which includes the dilatation term based on thermodynamics of irreversible processes. He formulated the theory of coupled thermoelasticity to eliminate the paradox inherent in the classical uncoupled theory that elastic changes have no effect on the temperature. The coupling between thermal and strain fields gives rise to the coupled theory of thermoelasticity.

Hetnarski [6] applied the methods of asymptotic expansions valid for, small values of time to obtain the solution for a thermal shock half-space problem and also solved a spherical symmetric problem with a point source of heat in the context of coupled thermoelasticity.

The equations of coupled thermoelasticity consist of two equations: the first, governing the displacement vector, is a wave type equation; the other, governing the temperature field, is a diffusion type equation. Due to the nature of the latter equation, if the elastic medium extending to infinity is subjected to mechanical or thermal disturbance, the effect will be felt instantaneously at infinity; this implies that a part of disturbance has an infinite velocity of propagation which is physically impossible. This paradox in the

\footnotetext{
* To whom correspondence should be addressed
} 
existing coupled theory of thermoelasticity has also been discussed by Boley [7]. To overcome this drawback need was felt to develop the theories of generalized thermoelasticity.

The first is due to Lord and Shulman [8], who introduced the theory of generalized thermoelasticity with one relaxation time by postulating a new law of heat conduction to replace the classical Fourier law. This law contains the heat flux vector as well as its time derivative.

The second generalization of the coupled theory of thermoelasticity is what is known as the theory of thermoelasticity with two relaxation times or the theory of temperature-rate-dependent thermoelasticity. Müller [9] in a review of thermodynamics of thermoelastic solids proposed an entropy production inequality, with the help of which, he considered restrictions on a class of constitution equations. A generalization of this inequality was proposed by Green and Laws [10]. Green and Lindsay obtained an explicit version of the constitutive equations in [11].

Dhaliwal and Sherief [12] derived the governing field equations of generalized thermoelasticity for anisotropic media and also developed a variational principle for these equations. Wilms and Cohen [13] discussed some one-dimensional problems in coupled thermoelasticity.

Green and Naghdi [14] proposed a new theory of thermoelasticity without energy dissipation and presented the derivation of a complete set of governing equations of the linearized version of the theory for homogenous and isotropic materials in terms of displacement and temperature fields and proved the uniqueness of the solutions of the corresponding initial mixed boundary value problem. An important feature of this theory, which is not present in other theories, is that this theory does not accommodate dissipation of thermal energy.

Dolotov and Kill [15] obtained an exact solution in closed form of the coupled dynamic problem of thermoelasticity for a half-space with a boundary condition of the first kind. They investigated the normal stress, perpendicular to the free surface, in the neighbourhood of the elastic wave front. Tzou [16] and Chandrasekharaiah [17] developed dual-phase-lag thermoelastic models, In these models two different phase-lags, i.e., one for the heat flux vector and other for the temperature gradient have been introduced in the Fourier's law.

Hetnarski and Ignaczak [18] in their survey article examined five generalizations of the coupled thermoelasticity, namely Lord and Shulman [8], Green and Lindsay [11], Green and Naghdi [14], Hetnarski and Ignaczak [19], Chandrasekharaiah and Tzou [17, 16], and obtained a number of interesting results.

Ishihara et al. [20] evaluated thermal stresses in an anisotropic material during thermal shock. Kumar and Rani [21] investigated the disturbance due to mechanical and thermal sources in a generalized orthorhombic thermoelastic material. Kumar and Rani [22] considered a two-diamensional problem of thermoelasticity and discussed the effects of mechanical and thermal sources in a generalized orthorhombic thermoelastic material. Weinmann [23] discussed equations of thermelasticity with time dependent coefficients. Sherief and El-Latief [24] applied the fractional order theory of thermoelasticity to a 2D problem for a half-space, solved it with the Laplace and exponential Fourier transform techniques and studied the effect of the fractional derivative parameter on the behavior of the solution. Sciarra and Salerno [25] discussed a consistent set of the thermodynamic functions by using the Green and Naghdi (GN) theory of thermoelasticity and derived free energy of GN, the thermodynamic potentials, i.e. internal energy, enthalpy and Gibbs free energy. Abbas et al. [26] studied ramp-type heating in a thermally conducting cubic crystal. Abbas [27] considered the problem of a thermoelastic infinite body with a spherical cavity in the context of the theory of fractional order thermoelasticity and discussed analytical solutions by using the eigen value approach and then graphically presented the effect of fractional parameters on physical quantities. El-Karamany and Ezzat [28] proposed three models of generalized thermoelasticity: a single phase - lag Green-Naghdi theory of type III, a dual-phase-lag Green-Naghdi theory of type II and of type III for a linear anisotropic inhomogeneous material. Abbas and Marin [29] established generalized thermoelasticity under laser pulse heating. Leseduarte et al. [30] discussed solutions for one-dimensional problem of thermoelasticity with two temperatures in the context of the Green-Lindsay and the LordShulman theories. They obtained existence and uniqueness of weakly regular solutions and proved the exponential stability in the Green-Lindsay model, but the non-exponential stability for the Lord-Shulman model. 
The present investigation is to determine the components of displacements, stresses and temperature distribution in a homogenous, thermally conducting twinned orthotropic thermoelastic material due to thermal loading in the form of triangular pulse. The problem investigated here has practical utility in the field of silicon based sensors, actuators, synthetic materials such as aircraft construction, fabric laminates, astronautics, designers and composite materials which are broadly used in civilian and military aircrafts.

\section{Basic equations}

Following Dhaliwal and Sherief [12], Lord and Shulman [8] and Choudhuri [31] the field equations and constitutive relations for homogeneous, anisotropic thermoelasticity in the absence of body forces and heat sources are given by

$$
\begin{aligned}
& t_{i j, j}=\rho \ddot{u}_{i} \\
& K_{i j}\left(1+\tau_{T} \frac{\partial}{\partial t}\right) \dot{T}_{, \mathrm{ij}}+K_{i j}^{*}\left(1+\tau_{v} \frac{\partial}{\partial t}\right) T_{, \mathrm{ij}}=\left(1+\tau_{q} \frac{\partial}{\partial t}+\tau_{q}^{2} \frac{\partial^{2}}{\partial t^{2}}\right)\left(\rho c_{e} \ddot{T}+\mathrm{T}_{\mathrm{o}} \beta_{i j} \ddot{u}_{i, j}\right), \\
& e_{k l}=s_{i j k l} \mathrm{t}_{\mathrm{ij}}+\alpha_{i j}\left(1+\tau_{a} \frac{\partial}{\partial t}\right) T_{, i}, \quad \alpha_{i j}=s_{i j k l} \beta_{k l}, \quad s_{i j k l}=\left(c_{k l i j}\right)^{-1} ; \quad(i, j, k, l=1,2,3)
\end{aligned}
$$

The comma notation is used for spatial derivatives and dot notation represents time differentiation. $s_{i j k l}$ satisfies the (Green) symmetry conditions

$$
s_{i j k l}=s_{k l i j}=s_{i j l k}=s_{j i k l} \text {. }
$$

Parameters in Eqs (2.1)-(2.3) are assumed to satisfy the following conditions:

(i) the thermal conductivity tensor $K_{i j}$ is symmetric and positive-definite;

(ii) the thermoelastic coupling tensor $\beta_{i j}$ is non-singular;

(iii) the specific heat $c_{e}$ at constant strain is positive;

(iv) the isothermal linear elasticities are positive-definite in the sense that

$$
s_{i j k l} e_{i j} e_{k l}>0
$$

\section{Formulation and solution of the problem}

We consider a homogenous, thermoelastic infinite crystal with orthotropic symmetry in the undeformed state at uniform temperature $T_{0}$. The infinite crystal is cut in two halves so that the plane interface contains one crystallographic axis (say $z$ ) and makes angle $\theta$ with another crystallographic axis (say $x$ ), see Fig.1a; then one rotate one half-space by 180 degrees about the normal to the interface, see Fig.1b; finally the half-spaces are rebonded, see Fig.1c. 


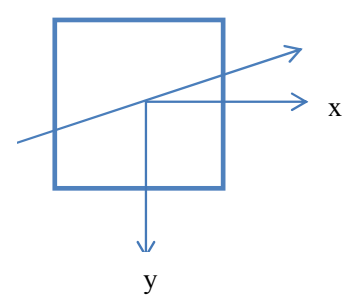

(a)

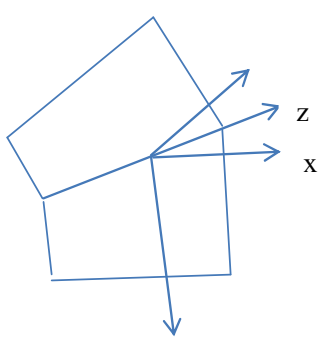

(c)

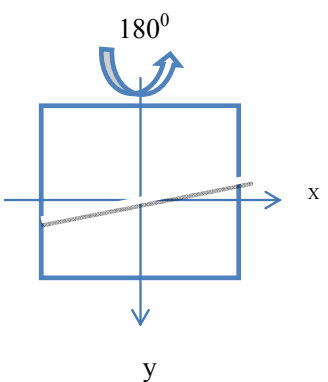

(b)

Fig.1. (a) Cutting, (b) rotating (c) bonding of an orthotropic crystal.

Another way of producing such twins would be as follows: consider the interface described in Fig.2, where the upper and lower half-spaces are made of the same crystal with at least orthotropic symmetry and with misoriented-crystallographic axes ' $x$ ' and ' $y$ ' (represented on the figure making an angle $\pm \theta$ with the interface and its normal) and common crystallographic axis $Z$ (normal to the plane in Fig.2).

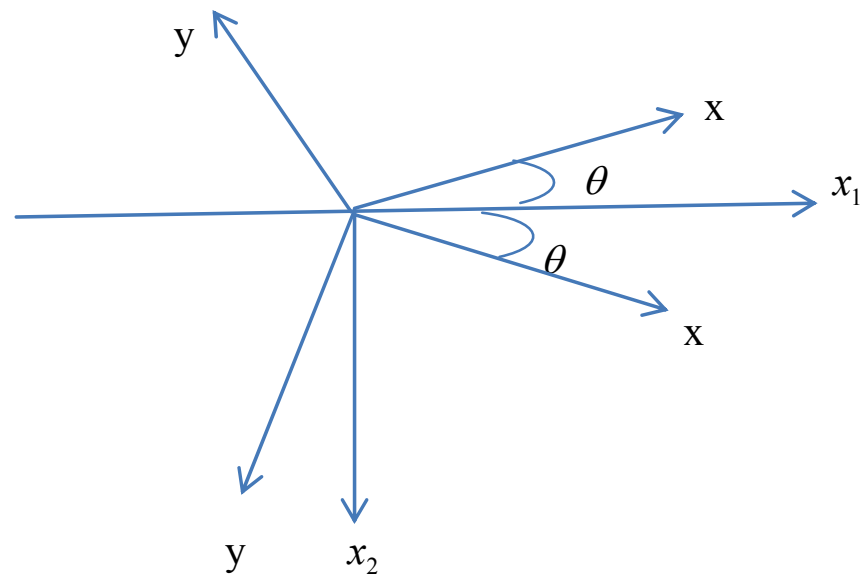

Fig.2. Twinned crystal.

The rectangular Cartesian co-ordinate system $(x, y, z)$ having its origin on the interface $y=0$ with the $y$-axis pointing into the medium is introduced. As an application, thermal loading in the form of a triangular pulse which depends on time $t$ and spatial coordinate $z(-\infty<z<\infty)$, is acting at some interior point of the infinite medium, taken as origin, i.e., the load is applied at the origin of the bi-material made of two perfectly bonded cubic crystal materials. Both half-spaces are made of the same crystal (mass density $\rho$ ). However, 
the normal to the interface $O x_{2}$ and the direction of propagation $O x_{1}$ are inclined at an angle $\theta$ to the crystallographic axis $o y$ and $o x$ of the lower $\left(x_{2}>0\right)$ half-space and at an angle - $\theta$ for the upper $\left(x_{2}<0\right)$ halfspace.

Following Ting [32] or Destrade [33], in the lower half-space isothermal elastic parameters are given as

$$
\begin{aligned}
& s_{11}=s_{11}^{\prime} \cos ^{4} \theta+\left(2 s_{12}^{\prime}+s_{66}^{\prime}\right) \cos ^{2} \theta \sin ^{2} \theta+s_{22}^{\prime} \sin ^{4} \theta, \\
& s_{22}=s_{22}^{\prime} \cos ^{4} \theta+\left(2 s_{12}^{\prime}+s_{66}^{\prime}\right) \cos ^{2} \theta \sin ^{2} \theta+s_{11}^{\prime} \sin ^{4} \theta, \\
& s_{12}=s_{12}^{\prime}+\left(s_{11}^{\prime}+s_{22}^{\prime}-2 s_{12}^{\prime}-s_{66}^{\prime}\right) \cos ^{2} \theta \sin ^{2} \theta, \\
& s_{66}=s_{66}^{\prime}+4\left(s_{11}^{\prime}+s_{22}^{\prime}-2 s_{12}^{\prime}-s_{66}^{\prime}\right) \cos ^{2} \theta \sin ^{2} \theta, \\
& s_{16}=\left[2 s_{22}^{\prime} \sin ^{2} \theta-2 s_{11}^{\prime} \cos ^{2} \theta+\left(2 s_{12}^{\prime}+s_{66}^{\prime}\right)\left(\cos ^{2} \theta-\sin ^{2} \theta\right)\right] \cos \theta \sin \theta, \\
& s_{26}=\left[2 s_{22}^{\prime} \cos ^{2} \theta-2 s_{11}^{\prime} \sin ^{2} \theta-\left(2 s_{12}^{\prime}+s_{66}^{\prime}\right)\left(\cos ^{2} \theta-\sin ^{2} \theta\right)\right] \cos \theta \sin \theta .
\end{aligned}
$$

In the upper half-space $\theta$ is changed to its opposite so that by Eqs (3.1) $s_{11}, s_{22}, s_{12}, s_{66}$ remains unchanged while $s_{16}, s_{26}$ change signs.

For the plane strain two-dimensional problem, we take a displacement vector

$$
\boldsymbol{u}=(u, v, 0)
$$

and $T(x, y, t)$ as temperature change.

Let us assume time harmonic behavior as

$$
(u, v, t)(x, y, t)=(u, v, t)(x, y) e^{i \omega t}
$$

where $\omega$ is the circular frequency.

Using the contracting subscript notations in Eq.(2.3) as $1 \rightarrow 11,2 \rightarrow 22$, $3 \rightarrow 33,4 \rightarrow 23,5 \rightarrow 13,6 \rightarrow 12$ to relate $s_{i j k l}$ to $s_{p q}(i, j, k, l=1,2,3$ and $p, q=1,2 \ldots \ldots, 6)$.

Making use of $s_{p q}$ from Eq.(2.3) in Eqs (2.1) and (2.2), then the field equations and constitutive relations for such a medium in the absence of body forces and heat sources in non-dimensional form after suppressing the primes can be rewritten as

$$
\begin{aligned}
& u,_{x x}+c_{l} u,_{y y}+c_{2} v,_{x y}-\beta_{l}\left(1+i \omega \tau_{a}\right) T,_{x}=-\rho \omega^{2} u, \\
& v,_{y y}+c_{3} v,_{x x}+c_{4} u,_{x y}-\beta_{2}\left(1+i \omega \tau_{a}\right) T,,_{y}=-\rho \omega^{2} v, \\
& \left(i \omega-\tau_{T} \omega^{2}\right)\left(T,_{x x}+T,,_{y y}\right)+\bar{K}\left(1 / \omega^{*}\right)\left(1+i \omega \tau_{v}\right)\left(T,_{x x}+T,,_{y y}\right)= \\
& =\left(-\omega^{2}\right)\left(1+i \omega \tau_{q}-\omega^{2} \frac{\tau_{q}^{2}}{2}\right)\left\{T+\in_{l}\left(u,_{x}+v,,_{y}\right)\right\}
\end{aligned}
$$


where comma notation is used for spatial derivatives. We have defined the quantities

$$
\begin{aligned}
& x^{\prime}=\frac{\omega_{1}^{*} x}{v_{1}}, \quad z^{\prime}=\frac{\omega_{1}^{*} z}{v_{1}}, \quad u^{\prime}=\frac{\rho v_{1} \omega_{1}^{*}}{\beta_{1} T_{0}} u, \quad t^{\prime}=\omega_{1}^{*} t, \quad v^{\prime}=\frac{\rho v_{1} \omega_{1}^{*}}{\beta_{1} T_{0}} v \\
& T^{\prime}=\frac{T}{T_{0}}, \quad c_{1}=\frac{s_{66}}{s_{11}}, \quad c_{2}=\frac{s_{12}+s_{66}}{s_{11}}, \quad c_{3}=\frac{s_{66}}{s_{22}}, \quad c_{4}=\frac{s_{12}+s_{66}}{s_{22}}, \quad \bar{K}=\frac{K^{*}}{K}, \quad \bar{\beta}=\frac{\beta_{2}}{\beta_{1}}, \\
& \omega^{\prime}=\frac{\omega}{\omega_{1}^{*}}, \quad \tau_{T}^{\prime}=\omega_{1}^{*} \tau_{T}, \quad \tau_{v}^{\prime}=\omega_{1}^{*} \tau_{v}, \quad t_{1}^{\prime}=\omega_{1}^{*} t_{1}, \quad \tau_{q}^{\prime}=\omega_{1}^{*} \tau_{q}, \quad \tau_{a}^{\prime}=\omega_{1}^{*} \tau_{a}, \quad \varepsilon_{1}=\frac{\beta_{1}^{2} T_{o}}{\rho k_{1} \omega_{1}^{*}}, \\
& t_{z z}^{\prime}=\frac{t_{z z}}{\beta_{1} T_{0}}, \quad t_{z x}^{\prime}=\frac{t_{z x}}{\beta_{1} T_{0}}, \quad h^{\prime}=\frac{h v_{1}}{\omega_{1}^{*}},
\end{aligned}
$$

and $v_{1}=\left(\frac{s_{11}}{\rho}\right)^{\frac{1}{2}}$ and $\omega_{1}^{*}=\frac{c_{e} s_{11}}{K_{1}}$ are, respectively, the velocity of compressional waves in the $x$-direction and characteristic frequency of the medium.

The initial and regularity conditions are given by

$$
\begin{aligned}
& u(x, y, 0)=0=\dot{u}(x, y, 0), \\
& w(x, y, 0)=0=\dot{w}(x, y, 0), \\
& T(x, y, 0)=0=\dot{T}(x, y, 0) \quad \text { for } y \geq 0, \quad-\infty<x<\infty,
\end{aligned}
$$

and $\quad u(x, y, t)=w(x, y, t)=T(x, y, t)=0$ for $t>0$ when $y \rightarrow \infty$.

Applying the Fourier transforms

$$
\tilde{f}(\xi, y, t)=\int_{-\infty}^{\infty} f(x, y, t) e^{i \xi x} d x
$$

on the resulting expressions, we obtain

$$
\begin{aligned}
& \frac{d^{2} \tilde{u}}{d y^{2}}=R_{11} \tilde{u}+R_{13} \frac{d \tilde{v}}{d y}+R_{15} \tilde{T} \\
& \frac{d^{2} \tilde{v}}{d y^{2}}=R_{22} \tilde{v}+R_{24} \frac{d \tilde{u}}{d y}+R_{26} \frac{d \tilde{T}}{d y}, \\
& \frac{d^{2} \tilde{T}}{d y^{2}}=R_{31} \tilde{u}+R_{33} \tilde{T}+R_{35} \frac{d \tilde{v}}{d y}
\end{aligned}
$$

where 


$$
\begin{aligned}
& R_{11}=\frac{\xi^{2}-\rho \omega^{2}}{c_{1}}, \quad R_{13}=\frac{i \xi c_{2}}{c_{1}}, \quad R_{15}=-\frac{i \xi \beta_{1}\left(1+i \omega \tau_{a}\right)}{c_{1}}, \\
& R_{22}=c_{3} \xi^{2}-\rho \omega^{2}, \quad R_{24}=i \xi c_{4}, \quad R_{24}=\beta_{2}\left(1+i \omega \tau_{a}\right), \\
& R_{31}=\frac{i \xi \varepsilon_{1} N_{1} \omega^{2}}{N_{2}}, \quad R_{33}=\frac{\xi^{2} N_{2}-\omega^{2} N_{1}}{N_{2}}, \quad R_{35}=-\frac{\varepsilon_{1} \omega^{2} N_{1}}{N_{2}}, \\
& N_{1}=\left(1+i \omega \tau_{q}-\omega^{2} \frac{\tau_{q}^{2}}{2}\right), \quad N_{2}=\left(i \omega-\tau_{T} \omega^{2}\right)+\frac{\bar{K}}{\omega_{1}^{*}}\left(1+i \omega \tau_{v}\right) .
\end{aligned}
$$

Equations (3.12)-(3.14) can be written as

$$
\frac{d}{d z}\{W(\xi, y, \omega)\}=A(\xi, \omega) W(\xi, y, \omega)
$$

where

$$
\begin{aligned}
W & =\left[\begin{array}{l}
U \\
U^{\prime}
\end{array}\right], \quad A=\left[\begin{array}{ll}
O & I \\
A_{1} & A_{2}
\end{array}\right], \quad U=\left[\begin{array}{l}
\tilde{u} \\
\tilde{v} \\
\tilde{T}
\end{array}\right], \quad U^{\prime}=\left[\begin{array}{l}
\tilde{u}^{\prime} \\
\tilde{v}^{\prime} \\
\tilde{T}^{\prime}
\end{array}\right], \\
O & =\left[\begin{array}{lll}
0 & 0 & 0 \\
0 & 0 & 0 \\
0 & 0 & 0
\end{array}\right], \quad I=\left[\begin{array}{ccc}
1 & 0 & 0 \\
0 & 1 & 0 \\
0 & 0 & 1
\end{array}\right], \quad A_{1}=\left[\begin{array}{lll}
0 & R_{15} & 0 \\
R_{24} & 0 & R_{26} \\
0 & R_{35} & 0
\end{array}\right], \\
A_{2} & =\left[\begin{array}{lll}
R_{11} & 0 & R_{13} \\
0 & R_{22} & 0 \\
R_{31} & 0 & R_{33}
\end{array}\right],
\end{aligned}
$$

To solve Eq.(3.15), we take

$$
W(\xi, z, \omega)=X(\xi, \omega) e^{q z}
$$

so that

$$
A(\xi, \omega) W(\xi, z, \omega)=q W(\xi, z, \omega)
$$

which leads to an eigenvalue problem. The characterstic equation corresponding to the matrix $\mathrm{A}$ is given by

$$
\operatorname{det}[A-q I]=0,
$$

which on expansion leads to

where

$$
q^{6}-\lambda_{1} q^{4}+\lambda_{2} q^{2}-\lambda_{3}=0
$$

$$
\lambda_{1}=R_{15} R_{24}+R_{33}+R_{22}+R_{11}+R_{26} R_{35},
$$




$$
\begin{aligned}
& \lambda_{2}=R_{15} R_{24} R_{33}-R_{13} R_{24} R_{35}+R_{22} R_{33}+R_{11} R_{26} R_{35}-R_{31} R_{15} R_{26}+R_{11} R_{33}-R_{31} R_{13}+R_{11} R_{22}, \\
& \lambda_{3}=R_{22}\left(R_{11} R_{33}-R_{31} R_{13}\right) .
\end{aligned}
$$

The roots of Eq.(3.18) are $\pm q_{\ell}(\ell=1,2,3)$.

The eigenvalues of the matrix A are roots of Eq.(3.17). The eigenvector $X(\xi, \omega)$ corresponding to the eigenvalues $q_{\ell}$ can be determined by solving the homogeneous equation

$$
[A-q \rrbracket X(\xi, \omega)=0 .
$$

The set of eigenvectors $X_{\ell}(\xi, \omega),(\ell=1,2,3,4,5,6)$ may be obtained as

where

$$
X_{\ell}(\xi, \omega)=\left[\begin{array}{l}
X_{\ell 1}(\xi, \omega) \\
X_{\ell 2}(\xi, \omega)
\end{array}\right]
$$

$$
\begin{aligned}
& X_{\ell 1}(\xi, \omega)=\left[\begin{array}{l}
-\xi \\
a_{\ell} q_{\ell} \\
b_{\ell}
\end{array}\right], \quad X_{\ell 2}(\xi, \omega)=\left[\begin{array}{c}
-\xi q_{\ell} \\
a_{\ell} q_{\ell}^{2} \\
b_{\ell} q_{\ell}
\end{array}\right], \quad q=q_{\ell}, \quad \ell=1,2,3, \\
& X_{\ell_{a} 1}(\xi, \omega)=\left[\begin{array}{c}
-\xi \\
-a_{\ell} q_{\ell} \\
b_{\ell}
\end{array}\right], \quad X_{\ell_{\mathrm{a}} 2}(\xi, \omega)=\left[\begin{array}{c}
\xi q_{\ell} \\
a_{\ell} q_{\ell}^{2} \\
-b_{\ell} q_{\ell}
\end{array}\right], \quad \ell_{a}=\ell+3, \quad q=-q_{\ell}, \quad \ell=1,2,3 .
\end{aligned}
$$

where

$$
\begin{aligned}
& a_{\ell}=\frac{\left\{\left(\bar{\beta}-c_{2}\right) \xi^{2}-\omega^{2} \bar{\beta}-c_{1} \bar{\beta} q_{\ell}^{2}\right\}}{\Delta_{\ell}}, \\
& b_{\ell}=\frac{\left\{c_{1} q_{\ell}^{2} \xi-\left(\xi^{2}-\omega^{2}\right) \xi\right\}\left\{\left(c_{1} \xi^{2}-\omega^{2}\right)-q_{\ell}^{2}\left(c_{3}-c_{2} \bar{\beta}\right)\right\}-q_{\ell}^{2} c_{2} \xi\left\{\left(\xi^{2}-\omega^{2}\right)-c_{1} q_{\ell}^{2}\right\} \bar{\beta}-c_{2} \xi^{2}}{\xi \Delta_{\ell}} \\
& \Delta_{\ell}=i\left\{\left(c_{1} \xi^{2}-\omega^{2}\right)-\left(c_{3}-c_{2} \bar{\beta}\right) q_{\ell}^{2}\right\}, \quad \ell=1,2,3
\end{aligned}
$$

The solution of Eq.(3.15) is given by

$$
W(\xi, y, \omega)=\sum_{\ell=1}^{3}\left[B_{\ell} X_{\ell}(\xi, \omega) \exp \left(q_{\ell} y\right)+B_{\ell+3} X_{\ell+3}(\xi, \omega) \exp \left(-q_{\ell} y\right)\right]
$$

where $B_{\ell}(\ell=1,2,3,4,5,6)$ are arbitrary constants.

Thus Eq.(3.20) represents the solution of the general problem in the plane strain case of homogeneous thermoelasticity by employing the eigenvalue approach and therefore it can be applied to a broad class of problems in the Fourier transform. Displacements and temperature distribution that satisfy the regularity conditions (3.10) are given by 


$$
\begin{aligned}
& \tilde{u}(\xi, y, \omega)=-\xi\left(B_{4} e^{-q_{1} y}+B_{5} e^{-q_{2} y}+B_{6} e^{-q_{3} y}\right), \\
& \tilde{w}(\xi, y, \omega)=-\left(a_{1} q_{1} B_{4} e^{-q_{1} y}+a_{2} q_{2} B_{5} e^{-q_{2} y}+a_{3} q_{3} B_{6} e^{-q_{3} y}\right), \\
& \tilde{T}(\xi, y, \omega)=\left(b_{1} B_{4} e^{-q_{1} y}+b_{2} B_{5} e^{-q_{2} y}+b_{3} B_{6} e^{-q_{3} y}\right) .
\end{aligned}
$$

\section{Application}

\subsection{Dynamic thermoelastic case}

\section{1a. Thermoelastic interactions due to thermal source}

The boundary conditions at the plane surface are

$$
\begin{array}{ll}
t_{y y}=0, \quad t_{y x}=0, & \text { at } \quad z=0, \\
\frac{\partial T}{\partial z}(x, y=0)=r(x, t), & \text { for the temperature gradient boundary, } \\
\text { or } & \\
T(x, y=0)=r(x, t), & \text { for the temperature input boundary }
\end{array}
$$

where $\quad r(x, t)=\eta(x) F(t)$.

Applying the Fourier transforms defined by Eq.(3.11), we get

$$
r(\xi, \omega)=\tilde{\eta}(\xi) \tilde{F}(\omega) .
$$

Making use of Eqs (2.3), (3.7)-(3.10) in the boundary conditions given by Eq.(4.1) and with the help of Eqs (3.21)-(3.23), we obtain the expressions for displacement components, stresses and temperature distribution as

$$
\begin{aligned}
& \tilde{u}=-r(\xi, \omega) \frac{\xi \sum_{m=1}^{3} \Delta_{m}^{\prime \prime} e^{-q_{m} z}}{T_{0} \Delta_{2}^{*}}, \quad \tilde{w}=-r(\xi, \omega) \frac{\sum_{m=1}^{3} a_{m} q_{m} \Delta_{m}^{\prime \prime} e^{-q_{m} z}}{T_{0} \Delta_{2}^{*}}, \quad \tilde{t}_{z x}=r(\xi, \omega) \frac{\sum_{m=1}^{3} s_{m} \Delta_{m}^{\prime \prime} e^{-q_{m} z}}{T_{0} \Delta_{2}^{*}} \\
& \tilde{t}_{z z}=r(\xi, \omega) \frac{\sum_{m=1}^{3} p_{m} \Delta_{m}^{\prime \prime} e^{-q_{m} z}}{T_{0} \Delta_{2}^{*}}, \quad \tilde{T}=-r(\xi, \omega) \frac{\sum_{m=1}^{3} b_{m} \Delta_{m}^{\prime \prime} e^{-q_{m} z}}{T_{0} \Delta_{2}^{*}}
\end{aligned}
$$

where

$$
\begin{aligned}
& \Delta=\Delta_{1}^{*}+h \Delta_{2}^{*}, \\
& \Delta_{1}^{*}=p_{1}\left(m_{3} q_{2} b_{2}-m_{2} q_{3} b_{3}\right)+p_{2}\left(m_{3} q_{3} b_{3}-m_{3} q_{1} b_{1}\right)+p_{3}\left(m_{2} q_{2} b_{1}-m_{1} q_{2} b_{2}\right),
\end{aligned}
$$




$$
\begin{aligned}
& \Delta_{2}^{*}=p_{1}\left(m_{2} b_{3}-m_{3} b_{2}\right)+p_{2}\left(m_{3} b_{1}-m_{1} b_{3}\right)+p_{3}\left(m_{1} b_{2}-m_{2} b_{1}\right), \\
& \Delta_{1}^{\prime \prime}=p_{2} s_{3}-s_{2} p_{3}, \quad \Delta_{2}^{\prime \prime}=p_{2} s_{3}-s_{1} p_{3}, \quad \Delta_{3}^{\prime \prime}=p_{1} s_{2}-s_{1} p_{2}, \\
& s_{m}=\frac{\xi q_{\ell}\left(1+i a_{\ell}\right)}{s_{11}}, \quad p_{\ell}=\frac{i \xi_{12}+a_{\ell} q_{\ell}^{2} s_{11}-b_{\ell} \bar{\beta}\left(1+i \omega \tau_{a}\right)}{s_{11}}, \quad \ell=1,2,3 .
\end{aligned}
$$

On replacing $\Delta$ by $\left(\frac{\omega_{1}^{*} T_{0}}{V_{1}}\right) \Delta_{1}^{*}$ and $T_{0} \Delta_{2}^{*}$, respectively, we obtain the expressions for the temperature gradient boundary and temperature input boundary.

We set a triangular pulse $\quad \eta(x)= \begin{cases}a+x, & -a \leq x \leq 0 \\ a-x, & 0<x \leq a \\ 0, & |x|>a\end{cases}$

in Eq.(4.2). Using Eqs (3.7)-(3.8) and applying the Fourier transform defined by Eq.(3.11), we get

$$
\tilde{\eta}(\xi)=\left[2\left\{1-\cos \left(\frac{\xi c_{2} a}{\omega_{1}^{*}}\right)\right\} / \xi^{2}\right], \quad \xi \neq 0
$$

and instantaneous loading as

with

$$
F(t)=F_{0} \delta(t)
$$

$$
\tilde{F}(\omega)=F_{0}
$$

where $F_{0}$ is a constant representing the magnitude of constant temperature and $\delta(t)$ is the Dirac delta function.

\subsection{Quasi-static thermo-elastic case}

In the quasi-static thermoelastic case, the variation of temperature with time is sufficiently small, the influence of the inertia term in Eq.(2.1) may be neglected and we obtain the expressions for displacement components, stresses and temperature distribution given by Eq.(4.2) with following changed values

$$
R_{11}=\frac{\xi^{2}}{c_{1}}, \quad R_{22}=\frac{c_{1} \xi^{2}}{c_{3}}
$$

\subsection{Static thermo-elastic case}

In the static thermo-elastic case, the temperature field is constant in time and we obtain the expressions for displacement components, stresses and temperature distribution given by Eq.(4.2) with following changed values as 


$$
\begin{aligned}
& \rho=0, \quad R_{31}=R_{35}=0, \quad R_{33}=\frac{\xi^{2}}{\bar{k}_{2}}, \quad \bar{k}_{3}=\frac{k_{2}^{*}}{k_{1}^{*}}, \quad \lambda_{1}=R_{15} R_{24}+R_{33}+R_{22}+R_{11}, \\
& \lambda_{2}=\left(R_{15} R_{24}+R_{22}+R_{11}\right) R_{33}+R_{11} R_{22}, \quad \lambda_{3}=R_{22} R_{11} R_{33} .
\end{aligned}
$$

\section{Special cases}

Transformed solutions of Eq.(4.2) reduces to various models of thermoelasticity as:

(1) Classical thermoelastic model $-K^{*}{ }_{i j}=0$.

(2) Dual phase-lag-model of thermoelasticity $-K_{i j}>K_{i j}^{*}, K_{i j}^{*}=0$.

(3) Lord Shulman (L-S) model - $K_{i j}^{*}=0, \tau_{T}=0, \tau_{v}=0, \tau_{q}=\tau$.

(4) Green Lindsay (G-L) model $-K^{*}{ }_{i j}=0, \tau_{T}=0, \tau_{v}=0, \tau_{a} \geq \tau_{q}>0$.

(4) Coupled thermoelasticity (CT) model $-K^{*}{ }_{i j}=0, \tau_{T}=\tau_{q}=\tau_{v}=0$.

(5) Uncoupled thermoelasticity (UCT) model $-\varepsilon_{l}=0, K_{i j}^{*}=0, \tau_{T}=\tau_{q}=\tau_{v}=0$.

(6) Green-Nighdi (G-N) model (Type-1) $-K_{i j}^{*}=0$.

(7) Green-Nighdi (G-N) model (Type-II)- $K_{i j}<<K_{i j}^{*}, \tau_{T}=\tau_{q}=\tau_{v}=0$.

(8) Green-Nighdi (G-N) model (Type-III)- $\tau_{T}=\tau_{q}=\tau_{v}=0$.

\section{Particular cases:}

\subsection{Transversely isotropic materials}

This type of medium has only one axis of thermal and elastic symmetry. We take the $z$ axis along the axis of symmetry. Then the non-vanishing elastic and thermal parameters are

$$
s_{11}=s_{22}, \quad K_{1}=K_{2}, \quad \alpha_{1}=\alpha_{2} .
$$

\subsection{Cubic crystal}

For cubic crystals, the non-vanishing elastic and thermal parameters are

$$
s_{11}=s_{22}, \quad K_{1}=K_{2}=K, \quad \beta_{1}=\beta_{2}=\beta, \quad \alpha_{1}=\alpha_{2}=\alpha_{t} .
$$

\subsection{Isotropic media}

For isotropic materials every direction is a direction of elastic as well as thermal symmetry and the non-vanishing elastic and thermal parameters are

$$
\begin{aligned}
& s_{11}=s_{22}=\lambda+2 \mu, \quad s_{12}=\lambda, \quad s_{66}=\mu, \quad K_{1}=K_{2}=K_{1}^{*}=K_{2}^{*}=K, \\
& \alpha_{1}=\alpha_{2}=\alpha_{t}, \quad \beta_{1}=\beta_{2}=\beta=(3 \lambda+2 \mu) \alpha_{t} .
\end{aligned}
$$




\section{Inversion of the transforms}

To obtain the solution of the problem in the physical domain, we must invert the transforms in Eq.(4.2) for three phase lags. These expressions are functions of $z$, the parameters of Fourier transforms $\xi$, respectively, and hence are of the form $\tilde{f}(\xi, y, t)$. To get the function $f(x, z, t)$ in the physical domain, first we invert the Fourier transform using

$$
f(x, y, t)=\frac{1}{2 \pi} \int_{-\infty}^{\infty} e^{-i \xi x} \tilde{f}(\xi, y, t) d \xi=\frac{1}{\pi} \int_{0}^{\infty}\left(\cos (\xi x) f_{e}-i \sin (\xi x) f_{0}\right) d \xi
$$

where $f_{e}$ and $f_{0}$ are, respectively, even and odd parts of the function $\tilde{f}(\xi, y, t)$. The method for evaluating this integral, as described by Press et al. [34], involves the use of Romberg's integration with an adaptive step size. It also uses the results from successive refinements of the extended trapezoidal rule followed by extrapolation of the results to the limit when the step size tends to zero.

\section{Conclusion}

1. A specific problems of an orthotropic twins crystal subjected to triangular loading has been studied.

2. The Fourier transforms technique is applied to the basic equations to form a vector matrix differential equation, which is then solved by the eigen value approach.

3. The expressions for displacement components, stresses and temperature distribution for the dynamic case, quasi-static and static cases are deduced.

\section{Nomenclature}

$$
\begin{aligned}
e_{i j} & - \text { strain tensor } \\
K_{i j}^{*}=\frac{c_{e} s_{11}}{4} & - \text { the material characteristic constant of the theory } \\
s_{i j k l} & - \text { isothermal elastic parameters } \\
T_{0} & - \text { uniform temperature } \\
T(x, y, z, t) & - \text { temperature change } \\
t & - \text { time } \\
t_{i j} & - \text { stress tensor } \\
\boldsymbol{u}=(u, v, w) & - \text { displacement vector } \\
\alpha_{k l} & - \text { linear thermal expansion tensor } \\
\rho & - \text { density } \\
\tau_{T}, \tau_{v}, \tau_{q} \text { and } \tau_{a} & - \text { thermal relaxation times }
\end{aligned}
$$

\section{References}

[1] Duhamel J.M.C. (1837): Second memoire sur les phenomenes thermo-mecaniques. - J. de I'Ecole Polytechnique, vol.15, pp.1-15.

[2] Neumann F. (1855): Vorlesungen uber die Theorie der Elastizität der Festen Körpern. - Leipzig.

[3] Voigt W. (1910): Lehrbuch Kristall Physik. - Teubner.

[4] Jeffreys H. (1930): The thermodynamics of an elastic solid. - Proc. Cambr. Philos. Soc., vol.26, pp.101-106.

[5] Biot M.A. (1956): Thermoelasticity and irreversible thermodynamics. - J. Appl. Phys.,vol.27, pp.240-253. 
[6] Hetnarski R.B. (1961): Coupled one-dimensional thermal shock problem for small time. - Arch. Mech. Stos., vol.13, pp.259-306.

[7] Boley B.A. (1964): High Temperature Structures and Materials. - Oxford: Pergamon Press.

[8] Lord H.W. and Shulman Y. (1967): A generalized dynamical theory of thermoelasticity.- J. Mech. Phys. Solids, vol.15, pp.299-309.

[9] Müller I. (1971): The coldness, a universal function in thermoelastic bodies. - Arch. Rat. Mech. Anal., vol.41, pp.319-332.

[10] Green A.E and Laws N. (1972): On the entropy production inequality. - Arch. Rat. Mech. Anal, vol.54, pp.1753.

[11] Green A.E. and Lindsay K.A. (1972): Thermoelasticity. - J. Elasticity, vol.2, pp.1-7.

[12] Dhaliwal R.S. and Sherief H.H. (1980): Generalized thermoelasticity for anisotropic media. - Quart. of Applied Mathematics, vol.38, pp.1-8.

[13] Wilms E.V. and Cohen H. (1985): Some one-dimensional problems in coupled thermoelasticity. - Mechanics Research Communications, vol.12, No.1, pp.41-47.

[14] Green A.E. and Naghdi P.M. (1993): Thermoelasticity without energy dissipation. - J. Elasticity, vol.31, pp.189208.

[15] Dolotov M.V. ans Kill I.D. (1996): The coupled dynamic problem of thermoelasticity for a half-space. - Journal of Applied Mathematics and Mechanics, vol.60, No.4, pp.683-686.

[16] Tzou D.Y. (1995): A unified field approach for heat conduction from macro to micro-scales. - ASME J. Heat Transf., vol.117, pp.8-16.

[17] Chandrasekharaiah D.S. (1998): Hyperbolic thermoelasticity: A review of recent literature. - Appl. Mech. Rev., vol.51, pp.705-729.

[18] Hetnarski R.B. and Ignaczak J. (1999): Generalized thermoelasticity. - J. Thermal Stresses, vol.22, pp.451-476.

[19] Hetnarski R.B. and Ignaczak J.(1996): Solition-like waves in a flow temperature nonlinear thermoelastic solid. Int. J. Engng. Sci., vol.34, pp.1767-1787.

[20] Ishihara S., Goshima T., Iwawaki S., Shimizu M. and Kamiya S. (2002): Evaluation of thermal stresses induced in anisotropic material during thermal shock. - Journal of Thermal Stresses, vol.25, pp.647-661.

[21] Kumar R. and Rani L. (2004): Deformation due to mechanical and thermal sources in generalized orthorhombic thermoelastic material. - Sadhana, vol.29, pp.429-447.

[22] Kumar R. and Rani L. (2007): Disturbance due to mechanical and thermal sources in orthorhombic thermoelastic material. - IJAME, vol.12, pp.677-692.

[23] Weinmann (2009): Equations of thermelasticity with time dependent coefficients. - J. Math. Anal. Appl. vol.350, pp.81-99.

[24] Hany H., Sherief A.M. Abd El-Latief (2014): Application of fractional order theory of thermoelasticity to a 2D problem for a half-space. - Applied Mathematics and Computation, vol.248, pp.584-592.

[25] Sciarra F.M.D. and Salerno M. (2014): On thermodynamic functions in thermoelasticity without energy dissipation. - European Journal of Mechanics - A/Solids, vol.46, pp.84-95.

[26] Abbas I.A., Kumar R. and Rani L. (2015): Thermoelastic interaction in a thermally conducting cubic crystal subjected to ramp-type heating. - Applied Mathematics and Computation, vol.254, pp.360-369. 
[27] Abbas I.A. (2016): Eigenvalue approach to fractional order thermoelasticity for an infinite body with a spherical cavity. - Journal of the Association of Arab Universities for Basic and Applied Sciences, vol.20, pp.84-88.

[28] El-Karamany A.S. and Ezzat M.A. (2016): On the phase- lag Green-Naghdi thermoelasticity theories. - Applied Mathematical Modelling, vol.40, pp.5643-5659.

[29] Abbas I.A. and Marin M. (2017): Analytical solution of thermoelastic interaction in a half-space by pulsed laser heating. - Vol.87, pp.254-260.

[30] Leseduarte M.C., Quintanilla R. and Racke R. (2017): On (non-)exponential decay in generalized thermoelasticity with two temperatures. - Applied Mathematics Letters, in press, Accepted Manuscript, Available online 1 March 2017.

[31] Choudhuri S.S.R. (2007): On a thermoelastic three-phase-lag model. - J. Thermal Stresses, vol.30, pp.231-238.

[32] Ting T.C.T. (2000): Recent developments in anisotropic elasticity. - International Journal of Solids and Structures, vol.37, pp.401-409.

[33] Destrade M. (2003): Rayleigh waves in symmetry planes of crystals: explicit secular equations and some explicit wave speeds. - Mechanics of Materials, vol.35, pp.931-939.

[34] Press W.H., Teukolsky S.A., Vellerling W.T. and Flannery B.P. (1986): Numerical recipes. - Cambridge: Cambridge Univ. Press.

Received: September 3, 2017

Revised: June 18, 2018 